\title{
Formation and condensation of excitonic bound states in the generalized Falicov-Kimball model
}

\author{
Pavol Farkašovský \\ Institute of Experimental Physics, Slovak Academy of Sciences \\ Watsonova 47, 04353 Košice, Slovakia
}

\begin{abstract}
The density-matrix-renormalization-group (DMRG) method and the HartreeFock (HF) approximation with the charge-density-wave (CDW) instability are used to study a formation and condensation of excitonic bound states in the generalized Falicov-Kimball model. In particular, we examine effects of various factors, like the $f$-electron hopping, the local and nonlocal hybridization, as well as the increasing dimension of the system on the excitonic momentum distribution $N(q)$ and especially on the number of zero momentum excitons $N_{0}=N(q=0)$ in the condensate. It is found that the negative values of the $f$-electron hopping integrals $t_{f}$ support the formation of zero-momentum condensate, while the positive values of $t_{f}$ have the fully opposite effect. The opposite effects on the formation of condensate exhibit also the local and nonlocal hybridization. The first one strongly supports the formation of condensate, while the second one destroys it completely. Moreover, it was shown that the zero-momentum condensate remains robust with increasing dimension of the system.
\end{abstract}




\section{Introduction}

The formation and condensation of excitonic bound states of conduction-band electrons and valence-band holes belongs surely to one of the most exciting ideas of contemporary solid state physics. Although the excitonic condensation has been predicted a long time ago [1], no conclusive experimental proof of its existence has been achieved yet. The latest experimental studies of materials with strong electronic correlations showed however, that there are a few promising candidates for the experimental verification of the excitonic condensation. The first one is the mixed valence compound $T m S e_{0.45} T e_{0.55}$, where detailed studies of the pressure-induced semiconductor-semimetal transition indicate that excitons are created in a large number and condense below $20 \mathrm{~K}$ [2]. Moreover, the charge-density-wave (CDW) state observed in the layered transition-metal dichalcogenide $1 T-\mathrm{TiSe}_{2}$ was claimed to be of excitonic origin [3]. Quite recently, as a further candidate for the excitonic state, a quasi one-dimensional system $\mathrm{Ta}_{2} \mathrm{NiSe_{5 }}$ has raised and attracted much experimental attention [4. In this material the flat band was observed by the ARPES experiment, which was interpreted to be due to excitonic condensation. These results have stimulated further experimental and theoretical studies with regard to the formation and possible condensation of excitonic bound states of electron and holes in correlated systems. At present, it is generally accepted that the minimal theoretical model for a description of excitonic correlations in these material could be the Falicov-Kimball model [5] and its extensions [6 [15]. In its original form, the Falicov-Kimball model describes a two-band system of the itinerant $d$ electrons (with the nearest-neighbor $d$-electron hopping constant $t_{d}$ ) and the localized $f$ electrons that interact only via a local $f$ - $d$ Coulomb interaction $U$ :

$$
H_{0}=-t_{d} \sum_{\langle i, j\rangle} d_{i}^{+} d_{j}+U \sum_{i} f_{i}^{+} f_{i} d_{i}^{+} d_{i}+E_{f} \sum_{i} f_{i}^{+} f_{i}
$$

where $\alpha_{i}^{+}$and $\alpha_{i}$ are the creation and annihilation operators of spinless electrons in the $\alpha=\{d, f\}$ orbital at site $i$ and $E_{f}$ is the position of the $f$-level energy. In what 
follows we consider $t_{d}=1$ and all energies are measured in units of $t_{d}$.

Since the local $f$-electron number $f_{i}^{+} f_{i}$ is strictly conserved quantity, the $d-f$ electron coherence cannot be established in this model. This shortcoming can be overcome by including an explicit local hybridization $H_{V}=V \sum_{i} d_{i}^{+} f_{i}+f_{i}^{+} d_{i}$ between the $d$ and $f$ orbitals. However, the hybridization between $d$ and $f$ orbitals is not the only way to develop $d-f$ coherence. Analytical and numerical studies of Batista et al. [6,7] showed that a finite $f$-electron bandwidth $H_{t_{f}}=-t_{f} \sum_{<i, j>} f_{i}^{+} f_{j}$ also induces $d$ - $f$ coherence, and thus it can lead to an excitonic condensate even in the absence of direct $d-f$ hybridization. Later this model has been used extensively to describe different phases in the ground state and specially properties of the excitonic phase [8-14]. It was found that the ground state phase diagram exhibits very simple structure consisting of only four phases, and namely, the full $d$ and $f$ band insulator, the excitonic insulator, the CDW and the staggered orbital order. The excitonic insulator is characterized by a nonvanishing $\left\langle d^{+} f\right\rangle$ average. The CDW is described by a periodic modulation in the total electron density of both $f$ and $d$ electrons, and the staggered orbital order is characterized by a periodic modulation in the difference between the $f$ and $d$ electron densities.

An extension of this model with local hybridization between the $f$ and $d$ orbitals has been studied very recently in our work [15]. The numerical analysis of the excitonic momentum distribution $N(q)=\left\langle b_{q}^{+} b_{q}\right\rangle$ (with $b_{q}^{+}=(1 / \sqrt{L}) \sum_{k} d_{k+q}^{+} f_{k}$, where L denotes the number of lattice sites) showed that this quantity diverges for $q=0$, signalizing a Bose-Einstein condensation of preformed excitons. Moreover, it was found that the density of zero momentum excitons $n_{0}=\frac{1}{L} N(q=0)$ (as well as the total exciton density $n_{T}=\frac{1}{L} \sum_{q} N(q)$ ) depends strongly on the values of the Coulomb interaction $U$ and that already for relatively small values of $U(U \sim 4)$ the significant fraction of $n_{0} / n_{T} \sim 0.5$ excitons is in the zero-momentum state.

In the current paper we examine in detail how robust is the excitonic state against 
the changes of other model parameters. In particular, we consider effects of the nonzero f-electron hopping $H_{t_{f}}$, the non-local hybridization $H_{n o n}=\sum_{<i, j>} V_{i, j} d_{i}^{+} f_{j}+H . c$. and the increasing dimension of the system, which together, but also separately model more realistically the physical situation in real rare-earth compounds than the model considered in our previous paper $\left(t_{f}=0, E_{f}=0, D=1, H_{\text {non }}=0\right)$. The crucial role of these factors in a correct description of ground state properties of these materials has been already confirmed by previous analytical and numerical studies. Indeed, it was found, that the f-band (f-electron hopping) of the opposite parity than the dband, stabilizes the excitonic phase, but only in the dimension $D>1$ [6, 7], while the nonlocal hybridization (in the mean-field approximation) fully destroys the excitonic phase [16]. It should be noted that the comfirmation (refutation) of the last result by exact calculations is of crucial importance for a correct description of ground states properties of some rare earth systems and especially the mixed valence compounds, like $\mathrm{SmB}_{6}$ [17]. In these materials the local hybridization is forbidden and only the nonlocal one (with inversion symmetry) between the nearest-neighbour f and d orbitals, is allowed [18]. On the base of the above mentioned facts we expect the strong effects of these factors also on the formation and condensation of the excitonic bound states. Besides these factors, we examine also the influence of the f-level position on the density of $d-f$ excitons, since the simple parametrization between the f-level position and external pressure $E_{f} \sim p$, widely used in the literature [19] allows us to predict, at least qualitatively, the behaviour of this quantity with the external pressure.

\section{Results and discussion}

\section{$2.1 \quad$ DMRG results}

To examine effects of the above mentioned factors on the formation and condensation

of excitonic bound states in the generalized Falicov-Kimball model we have performed 
exhaustive DMRG studies of the model Hamiltonian $H=H_{0}+H_{t_{f}}+H_{V}+H_{\text {non }}$ for a wide range of the model parameters at the total electron density $n=n_{d}+n_{f}=1$. In all examined cases we typically keep up to 500 states per block, although in the numerically more difficult cases, where the DMRG results converge slower, we keep up to 1000 states. Truncation errors [20,21], given by the sum of the density matrix eigenvalues of the discarded states, vary from $10^{-6}$ in the worse cases to zero in the best cases.

Let us start a discussion of our numerical results for the case of $H_{\text {non }}=0$. The DMRG results for this case are summarized in Figs. 1-4. Fig. 1a shows the bahaviour
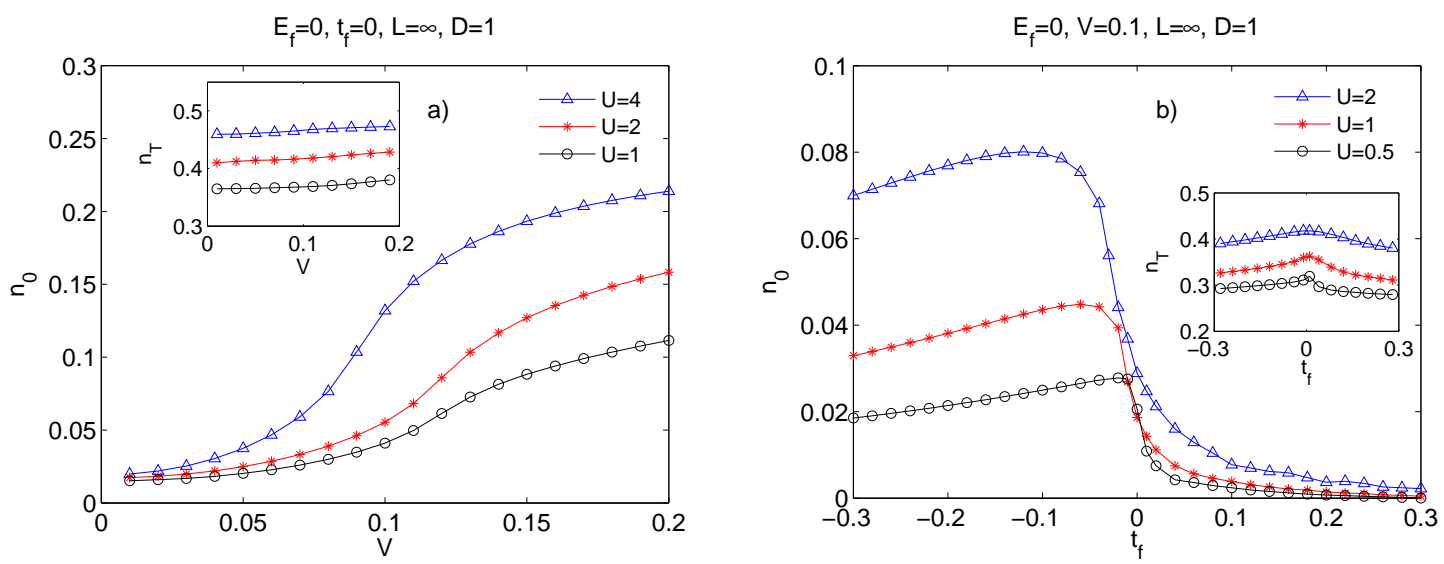

Figure 1: a) The density of zero-momentum excitons $n_{0}$ and the total exciton density $n_{T}$ as functions of local hybridization $V$ calculated by DMRG method for three different values of the interband Coulomb interaction $U\left(E_{f}=0, t_{f}=0, L=\infty, D=1\right)$. b) $n_{0}$ and $n_{T}$ as functions of $t_{f}$ calculated for three different values of $U\left(E_{f}=0, V=0.1, L=\infty, D=1\right)$.

of the density of zero momentum excitons $n_{0}$ as a function of local hybridization $V$ for several values of the interband Coulomb interaction $U$. One can see that both, the local interband hybridization $V$ as well as the local Coulomb interaction $U$ strongly support the condensation of preformed excitons to the zero-momentum state. In all examined cases the density of zero momentum excitons $n_{0}$ is a monotonically increasing function of $V$ which character changes obviously at some critical value of $V=V_{c}\left(n_{0} \sim V^{\nu}\right.$, with $\nu>1$ for $V<V_{c}$ and $n_{0} \sim V^{\nu}$, with $\nu<1$ for $V>V_{c}$ ). Comparing these results with ones obtained in our previous paper for the phase boundary between the CDW 
and homogeneous phase [15], the origin of this different behaviour is obvious. While below $V_{c}$ the excitonic phase coexists with the $C D W$ phase, above $V_{c}$ it coexists with the homogeneous one, what leads to a different power law behaviour of $n_{0}$ for $V<V_{c}$ and $V>V_{c}$. Unlike $n_{0}$, the total exciton density $n_{T}$ depends only very weakly on $V$ (see the inset in Fig. 1a) indicating that the formation of excitons is primarily driven by the interband Coulomb interaction $U$.

With regard to the situation in real materials, where always exists a finite overlap of $f$ orbitals on neighbouring sites, it is more interesting to ask what happens in the case when a finite $f$ bandwidth will be considered. In accordance with some previous theoretical studies, which documented strong effects of the parity of $f$ band on the stability of the excitonic phase [6,7] we have examined the model for both, the positive (the even parity) and negative (the odd parity) values of the $f$-electron hopping integrals $t_{f}$. The results of our non-zero $t_{f}$ DMRG calculations for $n_{0}$ are displayed in Fig. $1 \mathrm{~b}$ and they clearly demonstrate that the zero-momentum condensate is suppressed in the limit of positive values of $t_{f}$, while it remains robust for negative values of $t_{f}$. This result is intuitively expected since our previous Hartree-Fock (HF) results [22] showed that only the negative values of $t_{f}$ stabilize the ferroelectric phase, while the positive ones stabilize the antiferroelectric phase. The effect of $t_{f}$ is especially strong for $U$ small, where continuous but very steep changes of $n_{0}$ are observed for $t_{f} \rightarrow 0^{+}$. Contrary to this, the total exciton density $n_{T}$ exhibits only a weak dependence on the $f$-electron hopping parameter $t_{f}$, over the whole interval of $t_{f}$ values.

Till now we have presented results exclusively for $E_{f}=0$. Let as now discuss briefly the effect of change of the $f$-level position. This study is interesting also from this point of view that taking into account the parametrization between the external pressure and the position of the $f$ level $\left(E_{f} \sim p\right)$, one can deduce from the $E_{f}$ dependences of the ground state characteristics also their $p$ dependences, at least qualitatively [19]. The resultant $E_{f}$ dependences of the density of zero momentum excitons $n_{0}$ obtained by 
DMRG method are shown in Fig. 2a for several values of $V$ and $U=0.5$. One can see
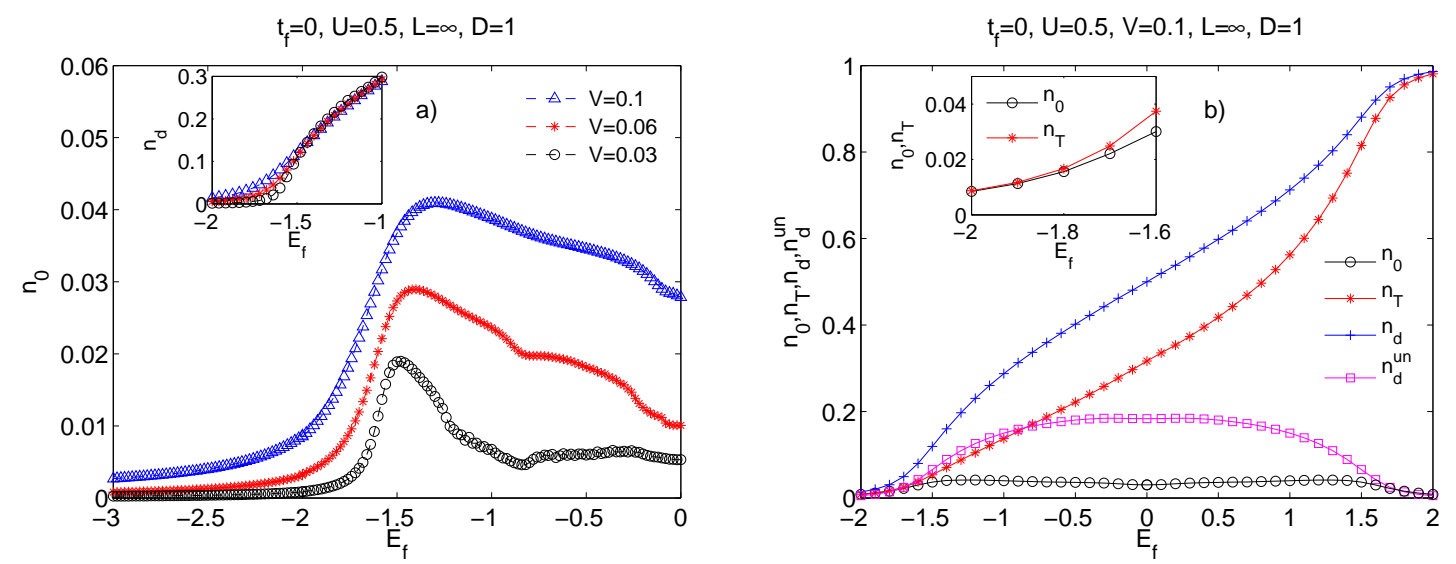

Figure 2: a) $n_{0}$ as a function of $E_{f}$ calculated by DMRG method for three different values of $V\left(t_{f}=0, U=0.5, L=\infty, D=1\right)$. The inset shows the density of $d$ electrons $n_{d}$ near $E_{f}=-1.5$. b) $n_{0}, n_{T}, n_{d}$ and $n_{d}^{u n}=n_{d}-n_{T}$ as functions of $E_{f}$ calculated for $t_{f}=0, U=$ $0.5, V=0.1, D=1$ and $L=\infty$. The inset shows the behaviour of $n_{0}$ and $n_{T}$ near $E_{f}=-2$.

that the density of zero momentum excitons is nonzero over the whole interval of $E_{f}$ values. Moreover, we have found that the values of $n_{0}$ are extremely enhanced in the region near $E_{f} \sim-1.5$, what is obviously due to the significant enhancement of the $d$ electron population in the $d$ band (see the inset in Fig. 2a).

To describe, in more detail, the process of formation of excitonic bound states with increasing $E_{f}$, we have plotted in Fig. 2b, besides the density od zero momentum excitons $n_{0}$, also the total exciton density $n_{T}$, the total $d$-electron density $n_{d}$ and the total density of unbond $d$ electrons $n_{d}^{u n}=n_{d}-n_{T}$. It is seen (see the inset in Fig. 2b) that below $E_{f} \sim-1.8, n_{0}$ and $n_{T}$ coincides what means that the excitonic insulator in this region is practically completely driven by the condensation of zeromomentum excitons. Above this value $n_{T}$ starts to increase sharply, while $n_{0}$ tends to its maximum at $E_{f} \sim-1.3$ and then gradualy decreases to its minimum at $E_{f}=0$. Similar behaviour with increasing $E_{f}$ exhibits also the density of unbond $d$ electrons $n_{d}^{u n}$, however the values of $n_{d}^{u n}$ are several times larger than $n_{0}$. It is interesting to note that although the total exciton density $n_{T}$ inreases over the whole interval of $E_{f}$ values, the number of unbond $d$ electrns remains practically uchanged over the 
wide range of $E_{f}$ values (from $E_{f}=-1$ to $E_{f}=1$ ), since its decrease, due to the formation of excitonic pairs, is compensated by the increase of $n_{d}\left(E_{f}\right)$. Thus we can conclude that in the pressure induced case, when the $f$-level energy shifts up with applied pressure [19], the model is able to describe, at least qualitatively, the increase in the total density of excitons with external pressure and the increase or decrease (according to the initial position of $E_{f}$ at ambient pressure) in the $n_{0}$ and $n_{d}^{u n}$.

As already mentioned, physically the most interesting case corresponds however to $H_{n o n}>0$. The importance of this term emphasizes the fact that the on-site hybridization $V$ is actually forbidden in real $d$ - $f$ systems for parity reasons. Istead the on-site hybridization, one has to consider in these materials the non-local hybridization with inversion symmetry $V_{i, j}=V_{n o n}\left(\delta_{j, i-1}-\delta_{j, i+1}\right)$ that leads to $k$-dependent hybridization of the opposite parity than corresponds to the $d$ band $\left(V_{k} \sim \sin (k)\right)[18$. A straightforward extension of the one-dimensional results to two dimensions yields $V_{i, j}=V_{n o n}\left[\delta_{i_{x}, j_{x}}\left(\delta_{i_{y}, j_{y}+1}-\delta_{i_{y}, j_{y}-1}\right)+\delta_{i_{y}, j_{y}}\left(\delta_{i_{x}, j_{x}+1}-\delta_{i_{x}, j_{x}-1}\right)\right]$, where any site on the lattice is given by $\mathbf{R}_{i}=i_{x} a \hat{\mathbf{x}}+i_{y} a \hat{\mathbf{y}}$ and $a$ is the lattice constant.

Typical examples of $1 / L$ dependence of the excitonic momentum distribution $N(q=$ 0) obtained for three representative values of the interband Coulomb interaction and two values of $f$-electron hopping in the one dimension are displayed in Fig. 3a and Fig. 3b. These results clearly demonstrate that there is no sign of divergence in the $1 / L$-dependence of $N(0)$ neither for $t_{f}=0$ nor for $t_{f}=-0.05$ and thus there is no signal of forming the Bose-Einstein condensate in the presence of non-local hybridization with the inversion symmetry. On the base of these results we can conclude that possible candidates for the appearance of the Bose-Einstein condensation of excitons in real $d-f$ materials, are only systems with local hybridization that supports the formation of the Bose-Einstein condensate, but not systems with non-local hybridization (of the inversion symmetry), what strongly limits the class of materials, where this phenomenon could be observed. On the other hand, it should be noted that although the 

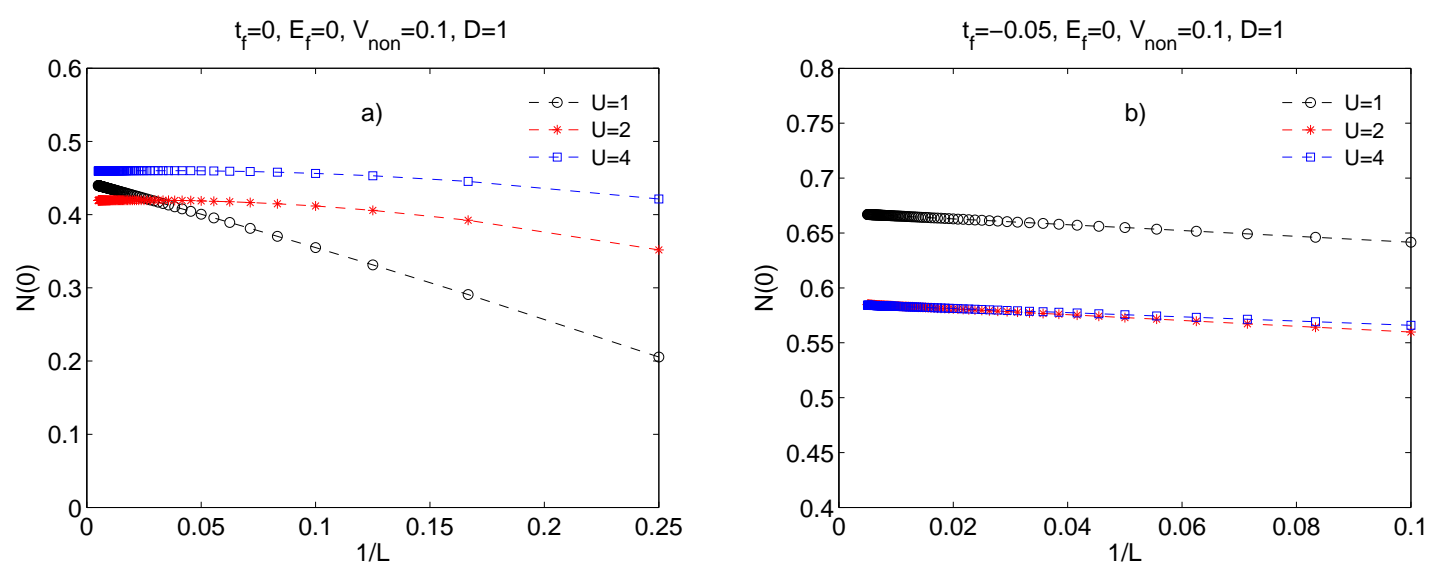

Figure 3: $N(0)$ as a function of $1 / L$ calculated by DMRG method for three different values of $U$ and two different values of $t_{f}\left(E_{f}=0, V_{\text {non }}=0.1\right)$.

local hybridization is forbidden in real $d-f$ electron systems, there is still a possibility to induce a finite local hybridization by some additional, not fully electronic mechanisms. Such an additional mechanism could be, for example, the electron-phonon interaction $H_{e l-p h}$. As shown by Menezes at al. 223. such an interaction can be reduced to the phonon-mediated local hybridization that can also lead to the formation of Bose-Einstein condensate under the supposition that it will be sufficiently strong, what depends on values of electron-phonon constants.

\section{$2.2 \quad$ Mean-field results}

Since not all of the above mentioned candidates are the one-dimensional or quasi onedimensional systems, it is interesting to ask if the above obtained results persist also in higher dimensions. Unfortunately, the DMRG method is not a very convenient tool for the study of lattice systems in $D>1$. Therefore, it is necessary to choose another method that should be able to describe sufficiently precise the excitonic effects in $d$ - $f$ systems. From this point of view a very promising method seems to be the HF approximation with the CDW instability. Indeed, we have showed in our previous paper [22], that this method is able to describe almost perfectly the ground-state phase diagram of the extended Falicov-Kimball model in $D=2$, including the existence of 
excitonic phase.

Let us briefly summarize the basic steps of this approximation. In the presence of CDW instability, the order parameters can be written as follows [16, 22]:

$$
\begin{gathered}
\left\langle n_{i}^{f}\right\rangle=n^{f}+\delta_{f} \cos \left(\mathbf{Q} \cdot \mathbf{r}_{i}\right), \\
\left\langle n_{i}^{d}\right\rangle=n^{d}+\delta_{d} \cos \left(\mathbf{Q} \cdot \mathbf{r}_{i}\right), \\
\left\langle f_{i}^{+} d_{i}\right\rangle=\Delta+\Delta_{P} \cos \left(\mathbf{Q} \cdot \mathbf{r}_{i}\right),
\end{gathered}
$$

where $\delta_{d}$ and $\delta_{f}$ are the order parameters of the CDW state for the $d$ - and $f$-electrons, $\Delta$ is the excitonic average and $\mathbf{Q}=\pi(\mathbf{Q}=(\pi, \pi))$ is the nesting vector for $D=1$ $(D=2)$.

Using these expressions the HF Hamiltonian of the generalized Falicov-Kimball model $\left(H=H_{0}+H_{t_{f}}+H_{V}+H_{n o n}\right)$ is

$$
\begin{aligned}
\mathcal{H} & =-t_{d} \sum_{\langle i, j\rangle} d_{i}^{+} d_{j}-t_{f} \sum_{\langle i, j\rangle} f_{i}^{+} f_{j}+E_{f} \sum_{i} n_{i}^{f}+U \sum_{i}\left(n_{f}+\delta_{f} \cos \left(\mathbf{Q} \cdot \mathbf{r}_{i}\right)\right) n_{i}^{d} \\
& +U \sum_{i}\left(n_{d}+\delta_{d} \cos \left(\mathbf{Q} \cdot \mathbf{r}_{i}\right)\right) n_{i}^{f}+\sum_{i j}\left(V_{i j}-U\left[\Delta+\Delta_{P} \cos \left(\mathbf{Q} \cdot \mathbf{r}_{i}\right)\right] \delta_{i j}\right) d_{i}^{+} f_{j}+H . c .
\end{aligned}
$$

This Hamiltonian can be diagonalized by the following canonical transformation

$$
\gamma_{k}^{m}=u_{k}^{m} d_{k}+v_{k}^{m} d_{k+\mathbf{Q}}+a_{k}^{m} f_{k}+b_{k}^{m} f_{k+\mathbf{Q}}, \quad m=1,2,3,4,
$$

where $\Psi_{k}^{m}=\left(a_{k}^{m}, b_{k}^{m}, u_{k}^{m}, v_{k}^{m}\right)^{T}$ are solutions of the associated Bogoliubov-de Gennes eigenequations:

$$
H_{k} \Psi_{k}^{m}=E_{k}^{m} \Psi_{k}^{m}
$$

with

$$
H_{k}=\left(\begin{array}{cccc}
\epsilon_{k}^{d}+U n_{f} & U \delta_{f} & V_{k}-U \Delta & -U \Delta_{P} \\
U \delta_{f} & \epsilon_{k+Q}^{d}+U n_{f} & -U \Delta_{P} & V_{k+Q}-U \Delta \\
V_{k}^{*}-U \Delta^{*} & -U \Delta_{P}^{*} & \epsilon_{k}^{f}+U n_{d}+E_{f} & U \delta_{d} \\
-U \Delta_{P}^{*} & V_{k+Q}^{*}-U \Delta^{*} & U \delta_{d} & \epsilon_{k+Q}^{f}+U n_{d}+E_{f}
\end{array}\right)
$$


and the corresponding dispersions $\epsilon_{k}^{d}, \epsilon_{k}^{f}$ and $V_{k}$ are obtained by the Fourier transform of the $d / f$-electron hopping amplitudes and the local/nonlocal hybridization. Then the HF parameters $n_{d}, \delta_{d}, n_{f}, \delta_{f}, \Delta, \Delta_{P}$ and the density of zero-momentum excitons $n_{0}$ can be expressed directly in terms of the Bogoliubov-de Gennes eigenvectors $a_{k}^{m}, b_{k}^{m}, u_{k}^{m}, v_{k}^{m}[16]$.

To verify the ability of this method to describe the formation of the Bose-Einstein condensate we have calculated firstly the density of the zero momentum excitons as functions of model parameters $V, t_{f}$ and $E_{f}$ for the one dimensional case. The results of our numerical calculations are displayed in Fig 4 (the left panels) and they show that the HF approximation with the CDW instability is able to decribe all aspects of formation of zero-momentum condensate in $D=1$ discussed above within the DMRG method. Although, there are some small qualitative as well as quantitative differences, like the discontinuous changes of $n_{0}$ with $t_{f}$ near $t_{f}=0$, or almost two times larger values of $V_{c}$ in comparison to DMRG studies (see the inset in the a) panel), in principle, the DMRG and HF pictures are same. In accordance with DMRG results we have found a strong enhancement of $n_{0}$ near $E_{f} \sim-1.5$ also in the HF solutions. Performing the numerical derivative of $n_{d}$ with respect to $E_{f}$ and comparing it with the behaviour of $n_{0}\left(E_{f}\right)$ one can easily verify (see the instet in the e) panel) that this enhancement is indeed connected with changes in the occupation of $f(d)$ orbitals as conjectured above on the base of $D M R G$ results.

The same calculations we have performed also in $\mathrm{D}=2$. The resultant behaviours of the density of zero momentum excitons $n_{0}$ as functions of $V, t_{f}$ and $E_{f}$ are plotted in Fig. 4 (the right panels) and they clearly confirm that with increasing dimension of the system the zero-momentum condensate remains robust. In comparison to the one-dimensional results there are two differences, and namely, that the discontinuous changes of $n_{0}$ (as a function of $t_{f}$ ) take place now strictly at $t_{f}=0$ in all examined cases and that the maximum in $n_{0}\left(E_{f}\right)$ shifts to higher values of $E_{f}$, where the periodic 
a) $E_{f}=0, t_{f}=0, D=1$

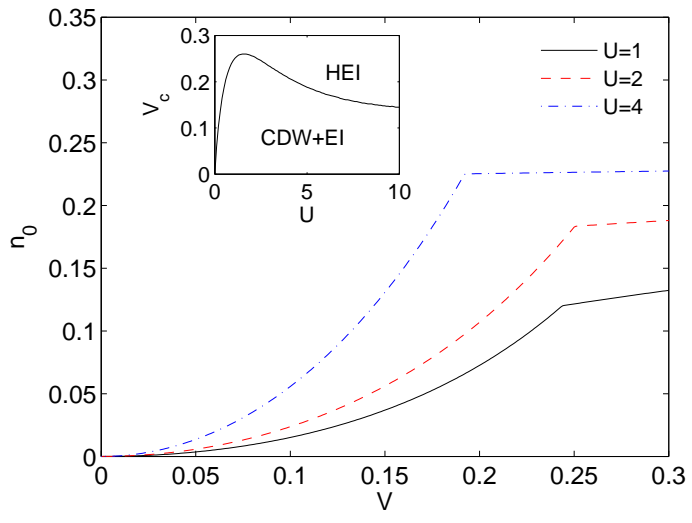

c) $E_{f}=0, V=0.1, D=1$

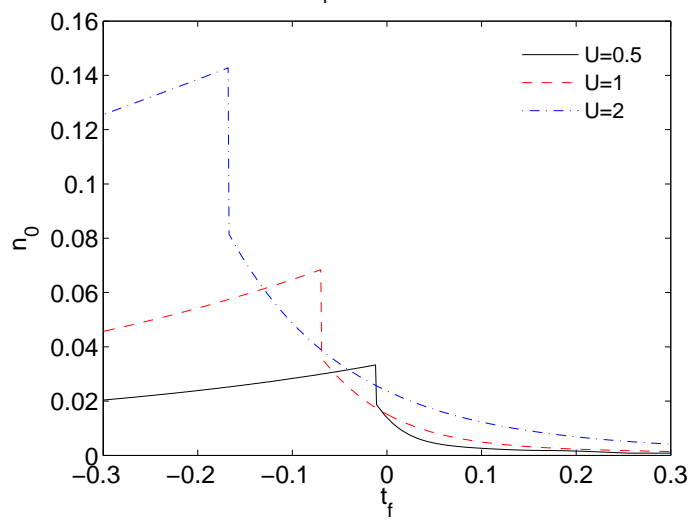

e) $t_{f}=0, U=0.5, D=1$

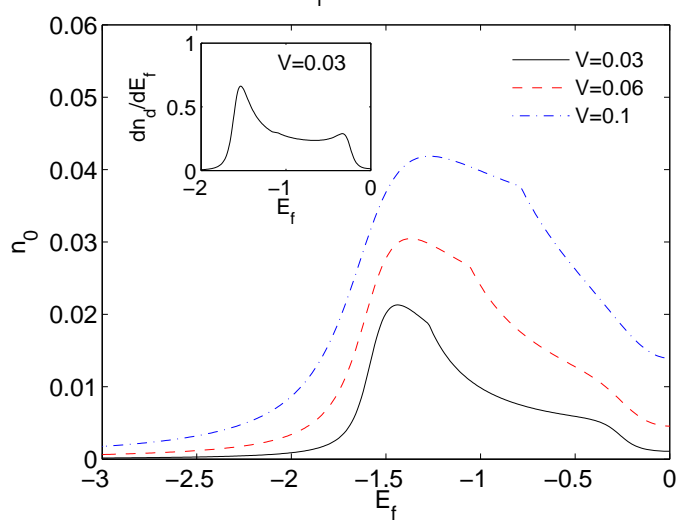

b) $E_{f}=0, t_{f}=0, D=2$

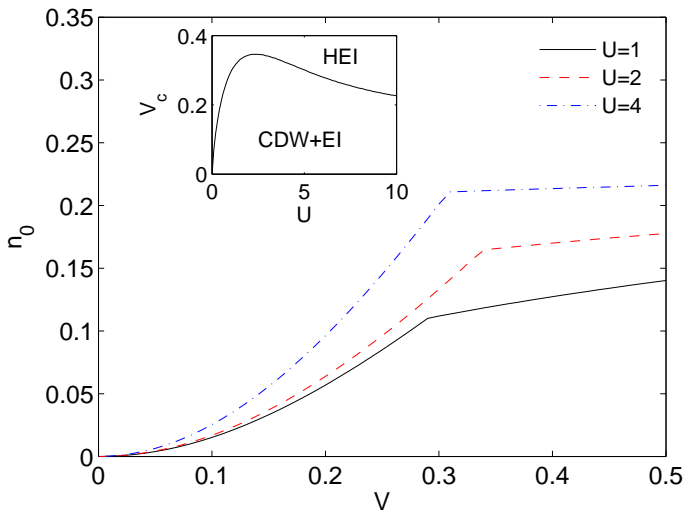

d) $E_{f}=0, V=0.1, D=2$

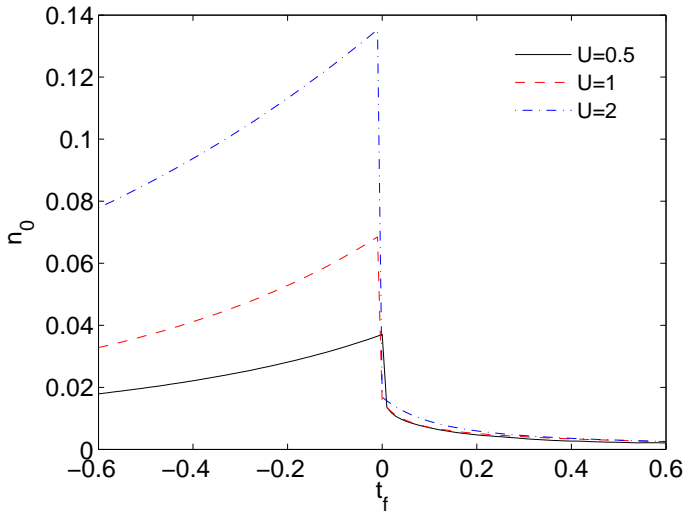

f) $t_{f}=0, U=0.5, D=2$

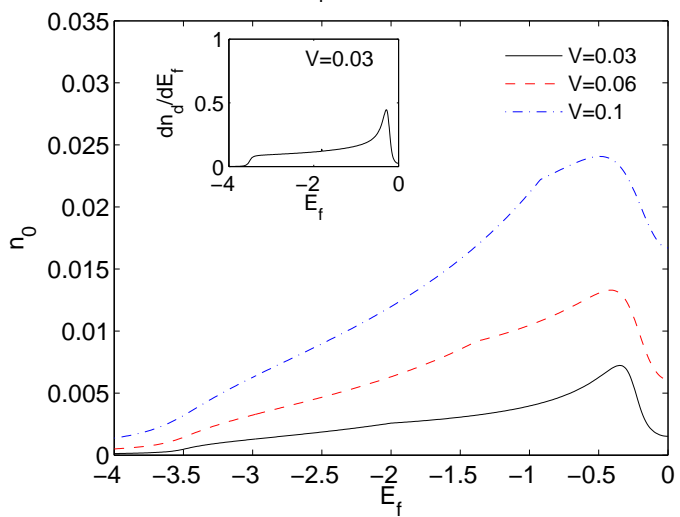

Figure 4: $n_{0}$ as a function of $V, t_{f}, E_{f}$ calculated by $\mathrm{HF}$ approximation with the $C D W$ instability in the one (the left panels) and two dimensions (the right panels). The insets in the panels a) and b) show the phase boundary between the homogeneous excitonic insulator (HEI) phase and the coexisting excitonic insulator and CDW state (CDW+EI).

solutions with CDW instability minimize the ground state energy. And finally, it should be noted that in accordance with DMRG results we have found no sign of formation of Bose-Einstein condensate for the case of nonlocal hybridization with the inversion 
symmetry neither in our HF solutions for both $D=1$ as well as $D=2$.

In conclusion, we have examined effects of various factors, like $f$-electron hopping, the local and nonlocal hybridization, as well as the increasing dimension of the system on the formation and condensation of excitonic bound states in the generalized Falicov-Kimball model. It was found that the negative values of the $f$-electron hopping integrals $t_{f}$ support the formation of zero-momentum condensate, while the positive values of $t_{f}$ have the fully opposite effect. The opposite effects on the formation of condensate exhibit also the local and nonlocal (with the inversion symmetry) hybridization. The first one strongly supports the formation of zero-momentum condensate, while the second one destroys it completely. Moreover, it was found that in the pressure induced case $\left(E_{f} \sim p\right)$, the model is able to describe, at least qualitatively, the increase in the total density of excitons $n_{T}$ with external pressure and the increase or deccrease (according to the initial position of $E_{f}$ at ambient pressure) in $n_{0}$ and $n_{d}^{u n}$. And finaly, it was shown (by HF studies) that the zero-momentum condensate remains robust with increasing dimension of the system.

This work was supported by Slovak Research and Development Agency (APVV) under Grant APVV-0097-12 and ERDF EU Grants under the contract No. ITMS 26220120005 and ITMS26210120002. 


\section{References}

[1] J. des Cloizeaux, J. Phys. Chem. Solids 26, 259 (1965); L.V. Keldysh and H.Y.V. Kopaev, Sov. Phys. Sol. State 6, 2219 (1965).

[2] J. Neuenschwander and P. Wachter, Phys. Rev. 41, 12693 (1990); B. Bucher, P. Steiner and P. Wachter, Phys. Rev. Lett. 67, 2717 (1991).

[3] C. Monney, G. Monney, P. Aebi, and H. Beck, Phys. Rev. B85, 235150 (2012); B. Zenker, H. Fehske, H. Beck, C. Monney, and A.R. Bishop, Phys. Rev. B88, 075138 (2013); G. Monney, C. Monney, B. Hildebrand, P. Aebi, and H. Beck, Phys. Rev. lett. 114, 086402 (2015); H. Watanabe, K. Seki, and S. Yunoki, Phys. Rev. B91, 205135 (2016).

[4] I. Wakisaka, T. Sudayama, K. Takubo, T. Mizokawa. M. Arita, H. Namatame, M. Taniguchi, N. Katayama, M. Nohara, and H. Takagi, Phys. Rev. Lett. 103, $026402(2009)$

[5] L.M. Falicov and J.C. Kimball, Phys. Rev. Lett. 22, 997 (1969).

[6] C. D. Batista, Phys. Rev. Lett. 89, 166403 (2002).

[7] C. D. Batista, J. E. Gubernatis, J. Bonca a H. Q. Lin, Phys. Rev. Lett. 92, 187601 (2004).

[8] B. Zenker, D. Ihle, F. X. Bronold, and H. Fehske, Phys. Rev. B 81, 115122 (2010).

[9] V. N. Phan, K. W. Becker, and H. Fehske, Phys. Rev. B 81, 205117 (2010).

[10] K. Seki, R. Eder, and Y. Ohta, Phys. Rev. B 84, 245106 (2011).

[11] B. Zenker, D. Ihle, F. X. Bronold, and H. Fehske, Phys.Rev. B 85, 121102R (2012). 
[12] T. Kaneko, K. Seki, and Y. Ohta, Phys. Rev. B 85, 165135 (2012).

[13] T. Kaneko, S. Ejima, H. Fehske, and Y. Ohta, Phys. Rev. B 88, 035312 (2013).

[14] S. Ejima, T. Kaneko, Y. Ohta and H. Fehske, Phys. Rev. Lett. 112, 026401 (2014).

[15] P. Farkašovský, EPL 110, 47007 (2015).

[16] P. M. R. Brydon, J. X. Zhu, M. Gulacsi, A. R. Bishop, Phys. Rev. B 72, 125122 (2005).

[17] I. Batko and M. Batkova, Solid State Commun. 196, 18 (2014); P. P. Baruselli and M. Vojta, Phys. Rev. Lett. 115, 156404 (2015).

[18] G. Czycholl, Phys. Rep. B 143, 277 (1986).

[19] C.E.T. Goncalves da Silva and L.M.Falicov, Solid State Commun. 17, 1521 (1975); P. Farkašovský, Phys. Rev. B 52, R5463 (1995); P. Farkašovský, Acta Physica Slovaca 60, 497 (2010).

[20] S. R. White, Phys. Rev. Lett. 69, 2863 (1992).

[21] A. L. Malvezzi, Braz. J. Phys. 33, 55 (2003).

[22] P. Farkašovský, Phys. Rev. B 77, 155130 (2008).

[23] F. Brouers and O. L. T. de Menezes, phys. stat. sol. 104, 541 (1981). 